\title{
Effect of Glottal Excitation Interchange in Hindi and Dogri Languages
}

\author{
Sonika Mahajan \\ Deptt of ECE, NITTTR \\ Chandigarh, India
}

\author{
Rajesh Mehra \\ Deptt of ECE, NITTTR \\ Chandigarh, India
}

\author{
Parveen K. Lehana \\ Department of Electronics \\ University of Jammu, India
}

\begin{abstract}
Voice acoustics is an active area of research which studies speaking voice and has gain popularity due to rapid advancements in digital signal processing. The shape of glottal excitation and the vocal tract may be speaker and language dependent. The objective of this paper is to study the effect of glottal excitation interchange on the quality and intelligibility in Hindi and Dogri languages. For this, recordings of six speakers (3 males and 3 females) were carried out in Dogri and Hindi languages. Cardinal vowels $(/ \mathrm{a} /, / \mathrm{i} /, / \mathrm{u} /)$ were extracted from recordings of each speaker. Investigations were carried out by interchanging the glottal excitations corresponding to the vowels in the two languages for each speaker. The analysis of the results showed that interchange of excitation does not provide satisfactory quality of the synthesized speech in terms of identity and clarity of speech. Further, the synthesized speech is perceived as it was spoken in the original language. It was also observed that if any two of the parameters (excitation, gain, vocal tract LPC coefficients) are interchanged, the accent of the original language also changes. It means that minimum two of the three parameters are necessary to interchange for modifying the accent of the language under consideration.
\end{abstract}

\section{General Terms}

Speech production, Linear predictive coding, LPC, Synthesizer

\section{Keywords}

LPC Component, vocal tract parameters, glottal source, glottal gain

\section{INTRODUCTION}

Speech is one of the most dominating and natural means of communication to express thoughts, ideas, and emotions among individuals [1]. Speech is a complicated signal, naturally produced by human beings, because various processes are involved in the generation of speech signal. Because of the involvement of several complicated processes, verbal communication may be modified extensively in terms of accent, pronunciation, articulation, nasality, pitch, volume, and speed [2]. Various anatomical articulators work in a synchronized manner for generating the meaningful speech. Slight variations in the movement of tongue, can directly affect the speech produced [3]. It is common to think of speech as involving two separate processes. The first one produces an initial sound called the excitation and the other one modifies it further due to change of the configuration of the vocal tract, which works as a filter. Speech is a sensation of air pressure vibrations produced by air exhaled from the lungs, modulated by the glottal cords, and filtered by the vocal tract. Speech has a rich and multilayered temporal spectral content encoding expression, accent, speaker identity, gender and age of the speaker. Speech may be broadly divided in to voiced and unvoiced segments. Voiced segments are produced because of the vibrations of the vocal folds. If the vocal folds do not vibrate, unvoiced segments in the speech are produced.

Speech generation may be understood with the help of speech production model shown in Fig 1. Here, the vocal tract filter is represented as $V(z)$ which models the transfer function relating the volume velocity at the lips to the glottal volume velocity. The input, $p(n)$, to the vocal tract is the differentiated glottal source signal and models the combined effects of the glottal volume velocity and the radiation effect at the lips. The output of the system is thus radiated sound pressure signal $s(n)$. This is called source filter model.

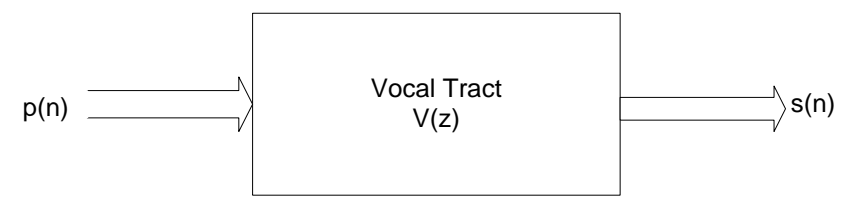

Fig 1: Speech production model

In the source filter model, it is assumed that the source is a spectrally flat excitation signal and the vocal tract filter is represented by the spectral envelope of speech. This representation of vocal tract which incorporates characteristics of the glottal signal may result into inaccuracies [4]. There are robust methods to extract the spectral envelope of speech, such as that used by STRAIGHT (Speech Transformation and Representation using Adaptive Interpolation of weight Spectrum) vocoder [4]. An important problem of source filter model is that it does not permit easy control of the glottal source. Similar problems arise with models using entire spectrum of the speech signal such as the Harmonicplus- Noise Model [5]. A widely used source filter model is linear predictive coding (LPC). LPC represent the speech waveform of time varying parameters which are related to the transfer function of the vocal tract and the characteristics of the source function [6].

Selection of the model depends upon the language under consideration. One model is generally not capable of representing multiple languages. As India is a linguistically diverse country with 22 official languages [7], there is a need of investigations of the relation of speech models and languages. Hindi is one of the prevalent languages in India after English and Mandarin. Hindi belongs to Devnagri script. Another similar language is Dogri, which is an Indo-Aryan language spoken by about five million people in India and Pakistan mainly in the Jammu region of Jammu and Kashmir. Dogri has its own script named as Doger. Number of Dogri speakers are far less than Hindi speakers. Hindi and Dogri are closely related languages having their roots in Sanskrit and belongs to the same subgroup of Indo -European family. In this paper the effect of glottal excitation interchange on the quality and intelligibility of speech in Hindi and Dogri languages is investigated. Investigations are carried out using LPC based analysis / synthesis platform. 


\section{LINEAR PREDICTIVE CODING}

Linear predictive coding (LPC) is widely used in speech synthesis and is capable of producing synthetic speech of high quality. LPC provides extremely accurate estimates of speech parameters. It is used for the extraction of the spectral envelope of speech in compact form [8]. The basic idea of LPC is that the current speech sample may be approximated as a linear combination of few past samples [9] [10].

$$
s(n)=\sum_{k=1}^{\rho} \alpha_{k} s(n-k)
$$

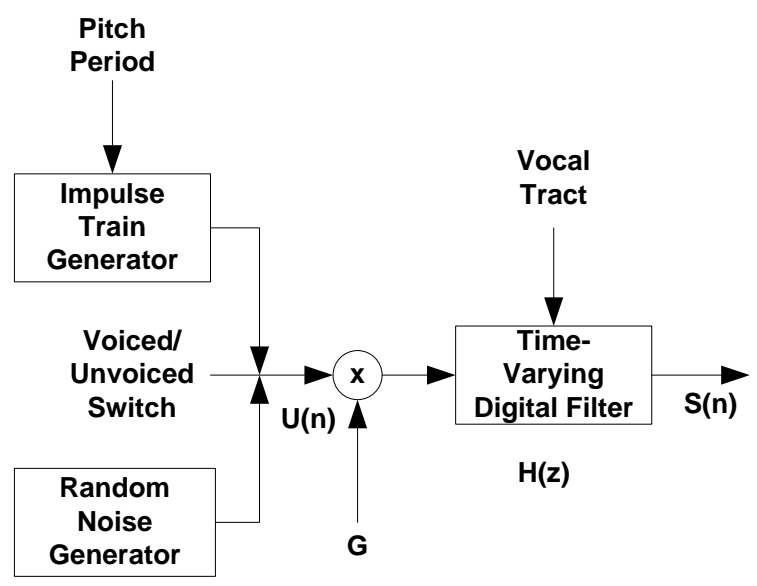

where $\rho$ is called the order and $\alpha_{\mathrm{k}}$ 's the LPC coefficients.

Fig. 2: Block diagram of an LPC synthesizer

A LPC based speech synthesizer is shown in Fig 2. The time varying all-pole digital filter, $H(z)$, is excited by periodic pulses for voiced speech and by white noise for unvoiced speech. The output of the filter $H(z)$ after appropriate digital to analog conversion and low pass filtering constitutes the synthetic speech signal. The predictor coefficients $\left(\alpha_{\mathrm{k}}\right.$ 's) are determined by minimizing the sum of squared differences between the actual speech samples and the linearly predicted.

\section{METHODOLOGY}

For the analysis and synthesis of the speech signal, speech of six speakers (3 males and 3 females) was recorded for sentences written in Hindi and Dogri languages at $16 \mathrm{kHz}$ sampling frequency and 16 bit quantization in an acoustically treated room using Sony ICD-AX-412 digital flash memory voice recorder. The speakers were able to speak fluently both Hindi and Dogri languages. Further, the speakers belonged to same age group. After recording, the speech was segmented manually and vowels $(/ a /, / i /, / u /)$ were extracted and investigations were conducted using four experiments. Experiment I was conducted for Hindi without any parameter modification. Similarly, Experiment II was conducted using Dogri without parameter modification.In Experiment III, glottal excitation was interchanged with excitation extracted from Dogri vowels. In Experiment IV, glottal excitation was interchanged with excitation from Hindi.

\section{RESULTS AND DISCUSSIONS}

Identity and clarity of each speaker for Experiments $\mathrm{I}$ is shown in Table I(a) and Table I(b) respectively. Similarly
Identity and Clarity of each speaker for Experiments II is shown in Table II(a) and Table II(b) respectively. Identity and Clarity of each speaker for Experiments III is shown in Table III(a) and Table III(b) respectively. Identity and Clarity of each speaker for Experiments IV is shown in Table IV(a) and Table IV(b) respectively. All of these experiments are conducted separately for each of the three vowels $(/ \mathrm{a} /, / \mathrm{i} /, / \mathrm{u} /)$. Histograms and spectrograms are also shown corresponding to these Experiments, for each of the three vowels. Mean (M) and Standard Deviation (SD) values are calculated using the observations of six listeners. It can be seen from the Mean values of Table I(a), Table I(b), Table II(a), Table II(b) that the clarity and identity of all the speakers is almost same as the original voice of the speakers. It can be seen from the Mean values of Table III(a), Table III(b), Table IV(a), Table IV(b), where glottal excitation is interchanged in Hindi and Dogri, that both the identity and clarity results of speakers are poor and unsatisfactory as in comparison with Experiments I and II. Similar observations can be done from the histograms.

\section{CONCLUSION}

The investigations were carried out to study the effect of glottal excitation interchange in Hindi and Dogri languages on the intelligibility and quality of the synthesized speech for three cardinal vowels using four experiments. The analysis of the results showed that LPC is able to synthesize Hindi and Dogri vowels with high quality, Table IV (b) Mean (M) and standard deviation (SD) values for experiment 4 in terms of clarity if the parameters are not modified. Interchange of excitation result in degradation of the quality and intelligibility. It was also observed that introduction of accent requires atleast two parameters interchange.

Table I (a) Mean (M) and standard deviation (SD) for Experiment $I$ in terms of identity

\begin{tabular}{|c|c|c|c|}
\hline Vowel & Speaker & M & SD \\
\hline \multirow[t]{6}{*}{ /a/ } & SP1 (F) & 5 & 0 \\
\hline & SP2 (F) & 5 & 0 \\
\hline & SP3 (F) & 5 & 0 \\
\hline & SP4 (M) & 5 & 0 \\
\hline & SP5 (M) & 5 & 0 \\
\hline & SP6 (M) & 5 & 0 \\
\hline \multirow[t]{5}{*}{ /i/ } & SP1 (F) & 5 & 0 \\
\hline & SP2 (F) & 5 & 0 \\
\hline & SP3 (F) & 5 & 0 \\
\hline & SP4 (M) & 5 & 0 \\
\hline & SP5 (M) & 5 & 0 \\
\hline
\end{tabular}




\begin{tabular}{|l|l|l|l|}
\hline \multicolumn{1}{|l|}{} & SP6 (M) & 5 & 0 \\
\cline { 2 - 4 } & SP1 (F) & 5 & 0 \\
SP2 (F) & 5 & 0 \\
SP3 (F) & 5 & 0 \\
SP4 (M) & 5 & 0 \\
SP5 (M) & 5 & 0 \\
SP6 (M) & 5 & 0 \\
\hline
\end{tabular}

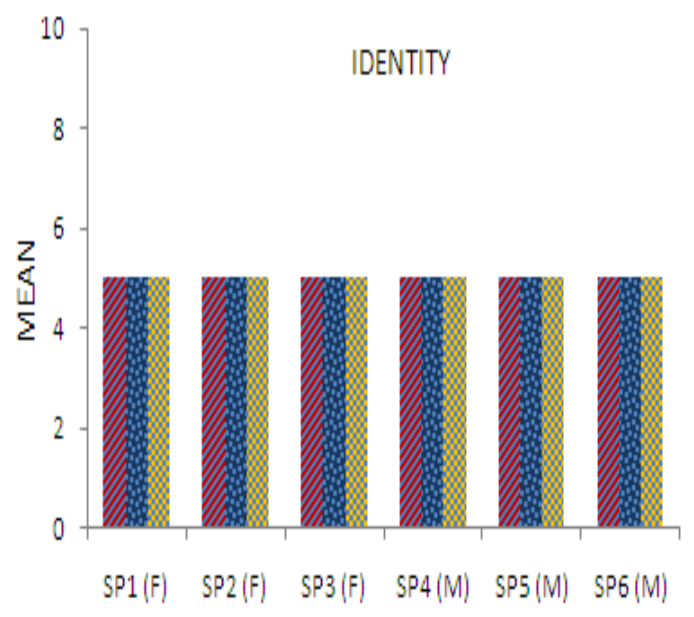

Fig 3: Comparison of mean of different speakers in terms of identity for Experiment I

\begin{tabular}{|c|c|c|c|}
\hline Vowel & Speaker & M & SD \\
\hline \multirow[t]{6}{*}{ /a/ } & SP1 (F) & 5 & 0 \\
\hline & SP2 (F) & 5 & 0 \\
\hline & SP3 (F) & 5 & 0 \\
\hline & SP4 (M) & 5 & 0 \\
\hline & SP5 (M) & 5 & 0 \\
\hline & SP6 (M) & 5 & 0 \\
\hline \multirow[t]{3}{*}{ /i/ } & SP1 (F) & 5 & 0 \\
\hline & SP2 (F) & 5 & 0 \\
\hline & SP3 (F) & 5 & 0 \\
\hline
\end{tabular}

\begin{tabular}{|l|l|l|l|}
\hline \multirow{1}{*}{$/ \mathrm{SP} 4(\mathrm{M})$} & 5 & 0 \\
$\mathrm{SP} 5(\mathrm{M})$ & 5 & 0 \\
$\mathrm{SP} 6(\mathrm{M})$ & 5 & 0 \\
\cline { 2 - 4 } & SP1 (F) & 5 & 0 \\
SP2 (F) & 5 & 0 \\
SP3 (F) & 5 & 0 \\
SP4 (M) & 5 & 0 \\
SP5 (M) & 5 & 0 \\
SP6 (M) & 5 & 0 \\
\hline
\end{tabular}

SP -Speaker, M- Mean, SD - Standard Deviation

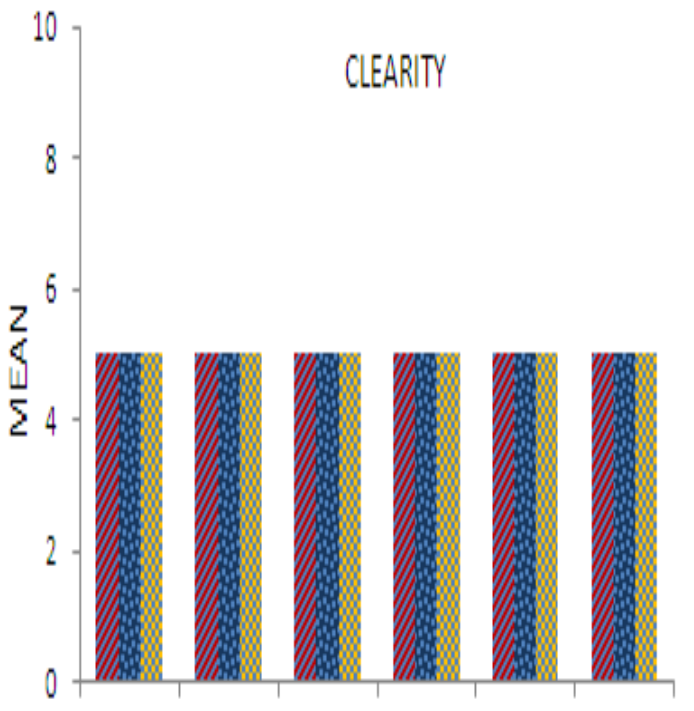

SP1/F) SP2(F) SPB(F) SP4(M) SP5/MI SP6/MI

Fig 4: Comparison of mean of different speakers in terms of Clarity for Experiment I

Table II. (a) Mean (M) and Standard Deviation (SD) for Experiment II in terms of identity

\begin{tabular}{|l|l|l|l|}
\hline Vowel & Speaker & M & SD \\
\hline /a/ & SP1 (F) & 5 & 0 \\
& SP2 (F) & 5 & 0 \\
& SP3 (F) & 5 & 0 \\
& SP4 (M) & 5 & 0 \\
& SP5 (M) & 5 & 0
\end{tabular}




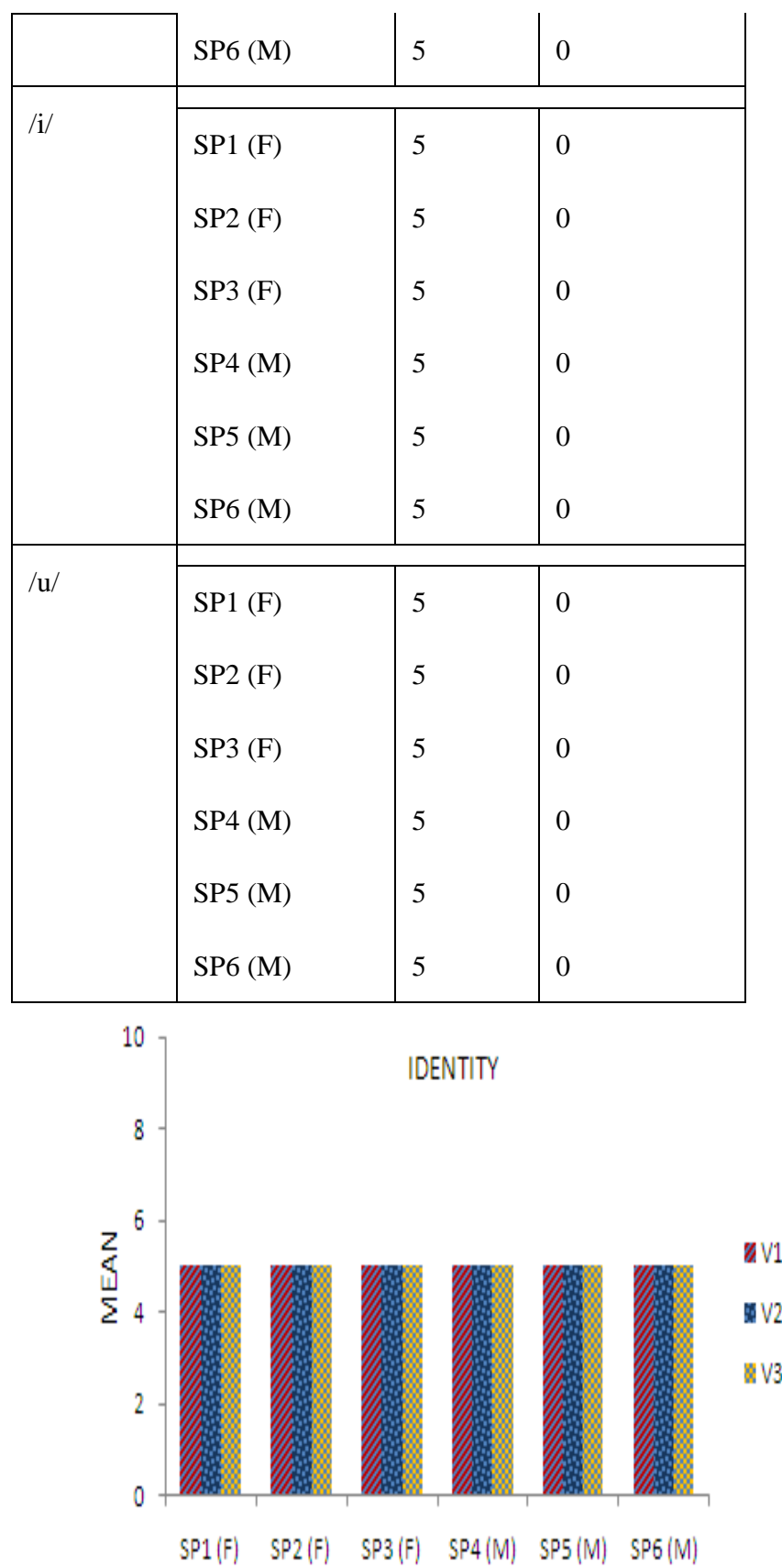

Fig 5: Comparison of mean of different speakers in terms of identity for Experiment II

\begin{tabular}{|l|l|l|l|}
\hline \multicolumn{4}{|c|}{ Table.II. (b) Mean (M) and standard deviation (SD) for } \\
experiment II in terms of clarity \\
\hline Vowel & Speaker & M & SD \\
\hline /a/ & SP1 (F) & 5 & 0 \\
SP2 (F) & 5 & 0 \\
SP3 (F) & 5 & 0 \\
\hline
\end{tabular}

\begin{tabular}{|l|l|l|l|}
\hline \multirow{1}{*}{$/$ SP5 (M) } & 5 & 0 \\
SP6 (M) & 5 & 0 \\
\cline { 2 - 4 } & \begin{tabular}{ll|l} 
SP1 (F) \\
SP2 (F)
\end{tabular} & 5 & 0 \\
SP3 (F) & 5 & 0 \\
SP4 (M) & 5 & 0 \\
SP5 (M) & 5 & 0 \\
SP6 (M) & 5 & 0 \\
\hline /u/ & SP1 (F) & 5 & 0 \\
SP2 (F) & 5 & 0 \\
SP3 (F) & 5 & 0 \\
SP4 (M) & 5 & 0 \\
SP5 (M) \\
SP6 (M)
\end{tabular}

SP -Speaker, M- Mean, SD - Standard Deviation

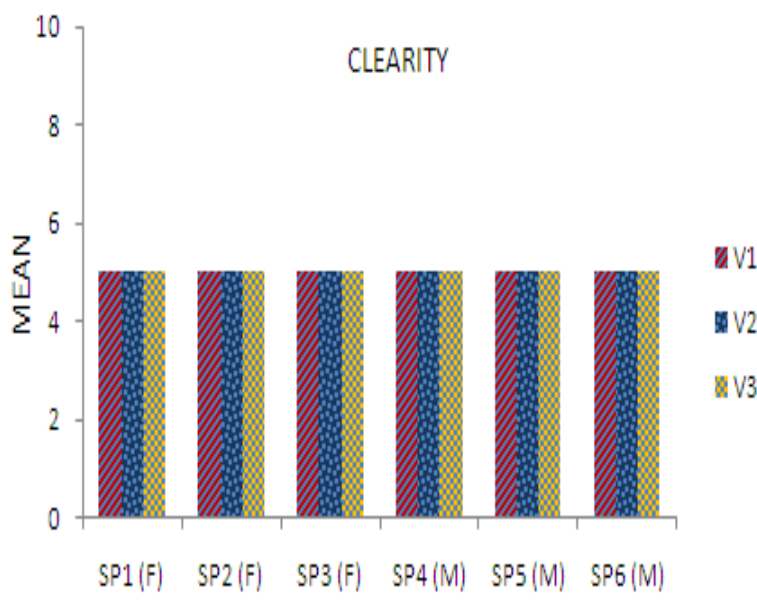

Fig 6: Comparison of mean of different speakers in terms of Clarity for Experiment II

Table III. (a) Mean (M) and standard deviation (SD) for Experiment III in terms of identity

\begin{tabular}{|l|l|l|l|}
\hline Vowel & Speaker & M & SD \\
\hline /a/ & SP1 (F) & 2.8 & 1.2 \\
& SP2 (F) & 3.2 & 1.1 \\
\cline { 2 - 4 }
\end{tabular}




\begin{tabular}{|c|c|c|c|}
\hline & $\begin{array}{l}\text { SP3 (F) } \\
\text { SP4 (M) } \\
\text { SP5 (M) } \\
\text { SP6 (M) }\end{array}$ & \begin{tabular}{l|}
3.2 \\
3.3 \\
3.2 \\
3.3
\end{tabular} & $\begin{array}{l}1.1 \\
1.2 \\
1.1 \\
1.2\end{array}$ \\
\hline /i/ & $\begin{array}{l}\text { SP1 (F) } \\
\text { SP2 (F) } \\
\text { SP3 (F) } \\
\text { SP4 (M) } \\
\text { SP5 (M) } \\
\text { SP6 (M) }\end{array}$ & $\begin{array}{l}3.2 \\
3.5 \\
3.3 \\
3.3 \\
3.3 \\
3.5\end{array}$ & $\begin{array}{l}1.1 \\
1.2 \\
1.2 \\
1.2 \\
1.2 \\
1.2\end{array}$ \\
\hline$/ \mathrm{u} /$ & $\begin{array}{l}\text { SP1 (F) } \\
\text { SP2 (F) } \\
\text { SP3 (F) } \\
\text { SP4 (M) } \\
\text { SP5 (M) } \\
\text { SP6 (M) }\end{array}$ & $\begin{array}{l}3.3 \\
3.2 \\
3.3 \\
3.3\end{array}$ & $\begin{array}{l}1.2 \\
1.1 \\
1.2 \\
1.2 \\
1.1 \\
1.1 \\
\end{array}$ \\
\hline
\end{tabular}

\begin{tabular}{|c|c|c|c|}
\hline & $\begin{array}{l}\text { SP3 (F) } \\
\text { SP4 (M) } \\
\text { SP5 (M) } \\
\text { SP6 (M) }\end{array}$ & $\begin{array}{l}3.5 \\
3.3 \\
3.7 \\
\\
3.7\end{array}$ & $\begin{array}{l}1.2 \\
1.2 \\
1.1 \\
1.1\end{array}$ \\
\hline$/ \mathrm{i} /$ & $\begin{array}{l}\text { SP1 (F) } \\
\text { SP2 (F) } \\
\text { SP3 (F) } \\
\text { SP4 (M) } \\
\text { SP5 (M) } \\
\text { SP6 (M) }\end{array}$ & \begin{tabular}{l|}
3.5 \\
\\
3.2 \\
3.2 \\
3.3 \\
3.2 \\
3.3
\end{tabular} & $\begin{array}{l}1.2 \\
1.1 \\
1.1 \\
1.2 \\
1.1 \\
1.2\end{array}$ \\
\hline$/ \mathrm{u} /$ & $\begin{array}{l}\text { SP1 (F) } \\
\text { SP2 (F) } \\
\text { SP3 (F) } \\
\text { SP4 (M) } \\
\text { SP5 (M) } \\
\text { SP6 (M) }\end{array}$ & $\begin{array}{l}3.2 \\
3.3\end{array}$ & $\begin{array}{l}1.1 \\
1.2\end{array}$ \\
\hline
\end{tabular}

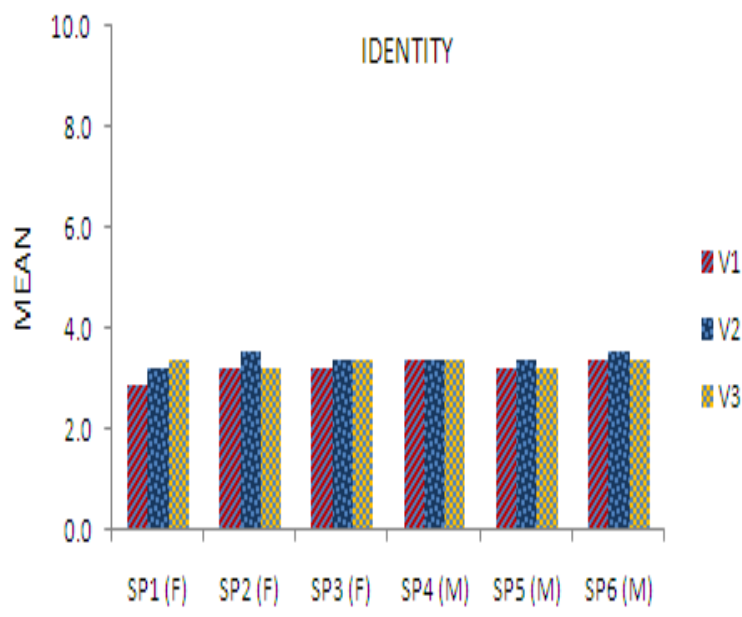

Fig.7: Comparison of mean of different speakers in identity for Experiment III

\begin{tabular}{|l|l|l|l|}
\hline \multicolumn{4}{|c|}{ Table III. (b) Mean (M) and Standard Deviation (SD) for } \\
Experiment III in terms of clarity
\end{tabular}

SP -Speaker, M- Mean, SD - Standard Deviation

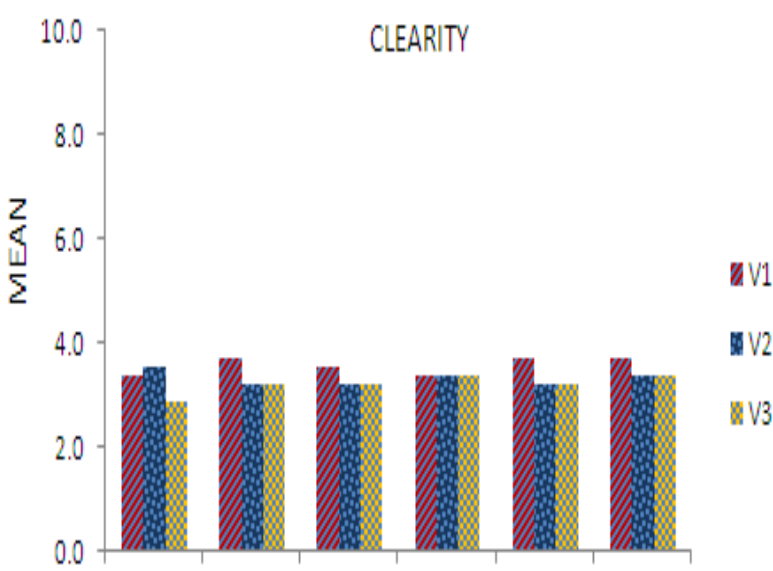

SP1(F) SP2(F) SP3(F) SP4(M) SP5(M) SPG (M)

Fig 8: Comparison of mean of different speakers in terms of Clarity for Experiment III

Table. IV(a): Mean (M) and standard deviation (SD) for Experiment IV in terms of identity

\begin{tabular}{|l|l|l|l|}
\hline Vowel & Speaker & M & SD \\
\hline$/$ a// & SP1 (F) & 2.7 & 1.4 \\
\hline
\end{tabular}




\begin{tabular}{|c|c|c|c|}
\hline & $\begin{array}{l}\text { SP2 (F) } \\
\text { SP3 (F) } \\
\text { SP4 (M) } \\
\text { SP5 (M) } \\
\text { SP6 (M) }\end{array}$ & $\begin{array}{l}3.3 \\
2.8 \\
3.2 \\
3.3 \\
3.3\end{array}$ & $\begin{array}{l}1.2 \\
1.2 \\
1.1 \\
1.2 \\
1.2\end{array}$ \\
\hline /i/ & $\begin{array}{l}\text { SP1 (F) } \\
\text { SP2 (F) } \\
\text { SP3 (F) } \\
\text { SP4 (M) } \\
\text { SP5 (M) } \\
\text { SP6 (M) }\end{array}$ & $\begin{array}{l}3.3 \\
3.7 \\
3.3 \\
3.5 \\
3.5 \\
3.3\end{array}$ & $\begin{array}{l}1.2 \\
1.1 \\
1.2 \\
1.2 \\
1.2 \\
1.2 \\
1.2\end{array}$ \\
\hline$/ \mathrm{u} /$ & $\begin{array}{l}\text { SP1 (F) } \\
\text { SP2 (F) } \\
\text { SP3 (F) } \\
\text { SP4 (M) } \\
\text { SP5 (M) } \\
\text { SP6 (M) }\end{array}$ & $\begin{array}{l}3.3 \\
3.3 \\
2.7 \\
2.8 \\
3.3\end{array}$ & $\begin{array}{l}1.2 \\
1.2 \\
1.4 \\
1.2 \\
1.2\end{array}$ \\
\hline
\end{tabular}

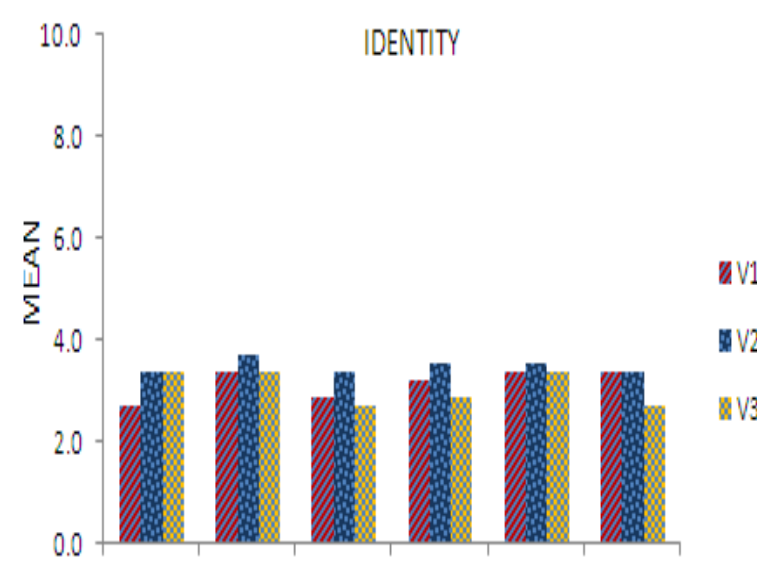

$\operatorname{SP1}(\mathrm{F}) \quad \mathrm{SP2}(\mathrm{F}) \quad \mathrm{SP3}(\mathrm{F}) \quad \mathrm{SP4}(\mathrm{M}) \quad \mathrm{SP5}(\mathrm{M}) \quad \mathrm{SPG}(\mathrm{M})$

Fig 9: Comparison of mean of different speakers in terms of identity for Experiment IV

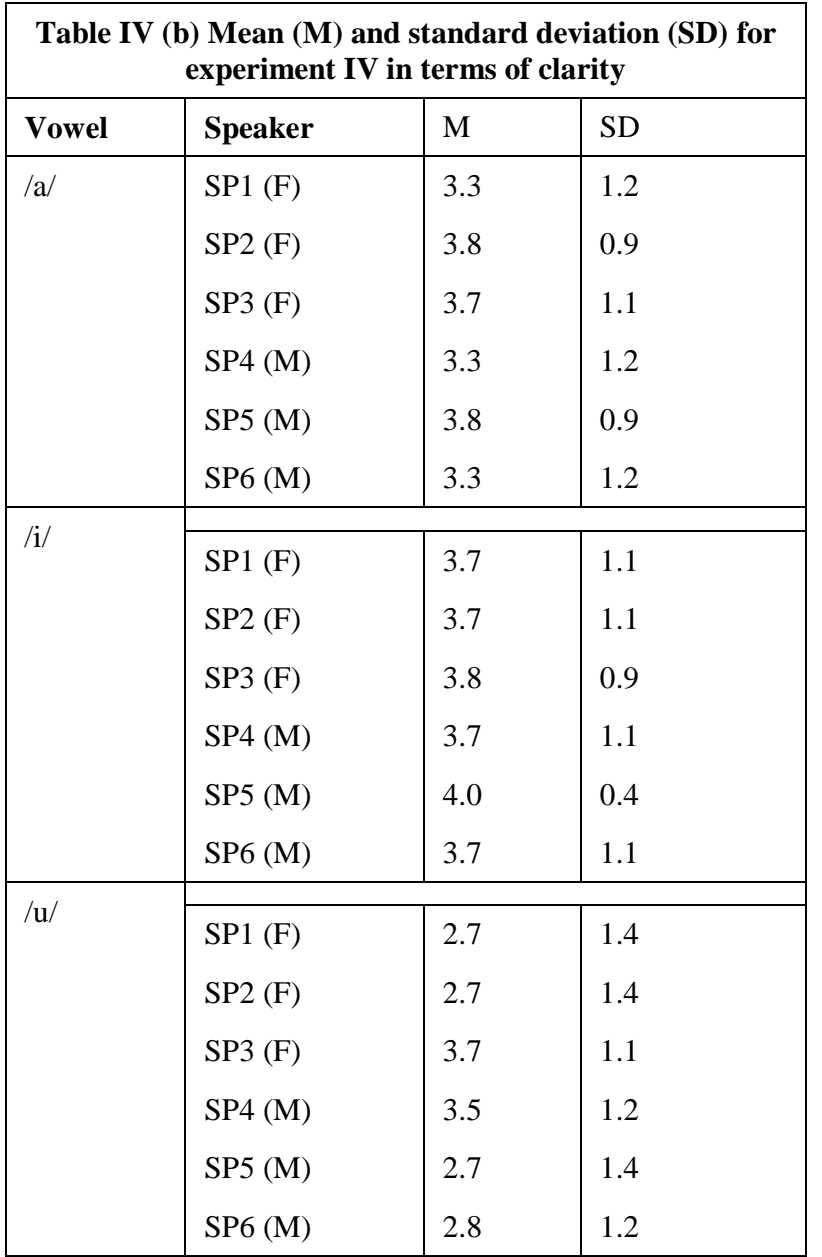

SP -Speaker, M- Mean, SD - Standard Deviation

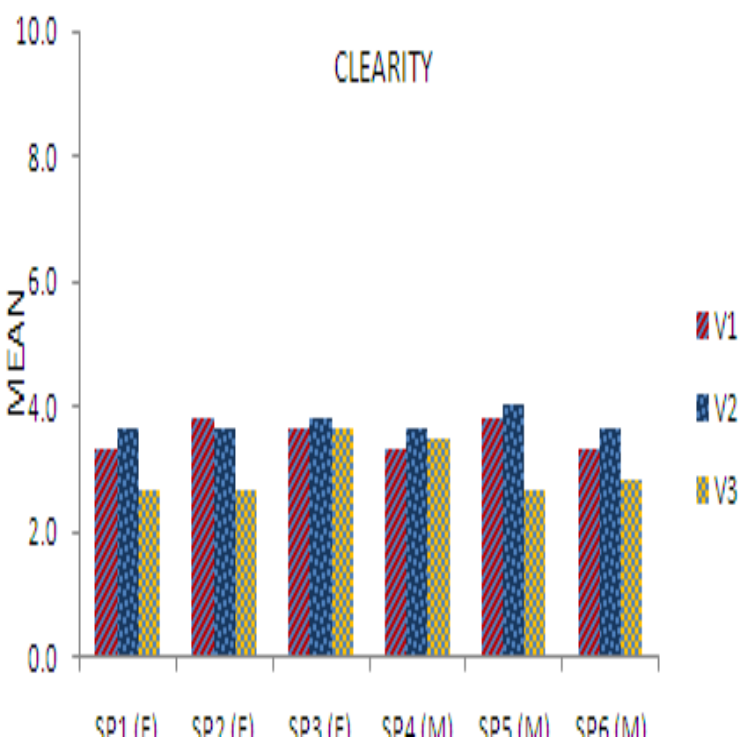

Fig.10: Comparison of mean of different speakers in terms of clarity for Experiment IV 

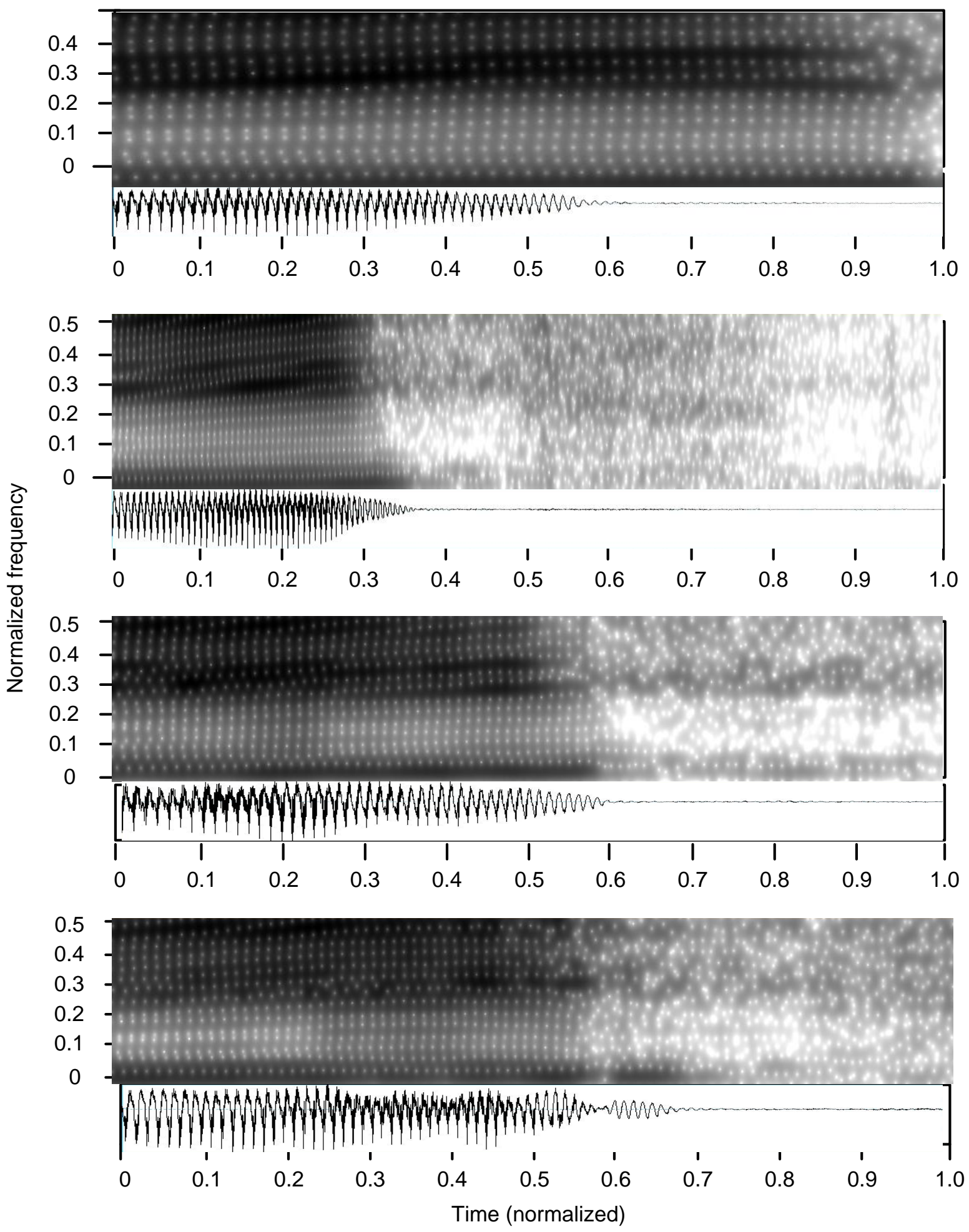

Fig 11: Spectograms obtained from recording of one of the speakers for vowel(/i/) a) Experiment I b) Experiment II c) Experiment III d) Experiment IV

\section{REFERENCES}

[1] Zaher AA. 2009. Recent Advances in signal processing. Publisher In Tech pp.544.

[2] Tebelskis J.1995. Speech Recognition using Neural Networks. Ph.D. desertation, Dept School of computer science Carnegie Mellon university Pitts burg,Pennsylvania.
[3] Gold Ben \& Morgan. N. 2000. Speech and Audio Signal Processing.Willey, New York.

[4] Joao P.Cabral,Korin Richmond, Junichi Yamagishi and Steve Renals. 2014. Glottal Spectral Separation for Speech Synthesis IEEE Journal, Vol. 8, No. 2, pp.195208.

[5] Kawahara,H.,Masuda-Katsuse,andA.Cheveign'e.1999. 
Restructuring speech representations using a pitch adaptive time- frequency smoothing and an instantaneous-frequency-based F0 Extraction :Possible role of a repetitive structure in sounds Speech Commun.,vol.27, no. 3-4, pp.187-207.

[6] Rulph Chassaing and Donald Reay. 2008. Digital Signal Processing and Applications with C6713 and C6416 DSK, 2ndEdition Wiley Inter Science Pub, London, IEEE PRESS.

[7] Dubey Preeti Pathania Shashi \& Devanand. 2011. Comparative study Hindi and Dogri languages with regard to machine translation language in India. Vol.11, pp.298-309.
[8] Padmini Rajput \& ParveenLehana. 2013. Investigations of the Distributions of Phonemic Durations in Hindi and Dogri.IJNLC Vol. 2, No.1, pp. 17-30.

[9] Ananthapadmanabha T.V. and B.Yegnarayanana. 1979. Epoch extraction from linear prediction residual for identification of closed glottis interval. IEEE Transactions on Acoustics, Speech, and Signal Processing, 27(4):309-319.

[10] Comeliu Octavian DUMITRU, Inge GAVAT. 2006.A Comparative Study of Feature Extraction Methods Applied to Continuous Speech Recognition in Romanian language.48thInternational Symposium, Zadar,Croatia. 